\title{
Going the distance for procurement of donation after circulatory death livers for transplantation-Does reimbursement reflect reality?
}

\author{
John R. Montgomery ${ }^{1}$ (D) | Alexandra Highet ${ }^{1}$ | Mark J. Hobeika² (i) | \\ Michael J. Englesbe $^{1}$ | Lisa M. McElroy ${ }^{1}$ (D)
}

${ }^{1}$ Department of Transplant Surgery, University of Michigan, Ann Arbor, Michigan

${ }^{2}$ Houston Methodist JC Walter Jr Transplant Center, Houston, Texas

\section{Correspondence}

John R. Montgomery, MD, Department of Surgery, University of Michigan, $1500 \mathrm{E}$.

Medical Center Drive, Ann Arbor, MI 48109.

Email: montgomj@med.umich.edu

Funding information

National Institute of Diabetes and Digestive and Kidney Diseases, Grant/Award Number: T32-DK108740

\begin{abstract}
Donation after circulatory death (DCD) liver transplantation (LT) has increased slowly over the past decade. Given that transplant surgeons generally determine liver offer acceptance, understanding surgeon incentives and disincentives is paramount. The purpose of this study was to assess aggregate travel distance per successful DCD versus deceased after brain death (DBD) liver procurement as a surrogate for surgeon time expenditure and opportunity cost. All consecutive liver offers made to Michigan Medicine from 2006 to 2017 were analyzed. Primary outcome was the summative travel distance (spent on all attempted procurements) per successful liver procurement that resulted in LT. Donation after circulatory death liver offer acceptance was lower than DBD liver offers, as was proportion of successful procurements among accepted offers. Overall, 10275 miles were travelled for accepted DCD liver offers, resulting in 23 successful procurements (mean 447 miles per successful DCD liver procurement). For accepted DBD liver offers, 197299 miles were travelled, resulting in 863 successful procurements (mean 229 miles per successful DBD liver procurement). On average, each successful DCD liver procurement required 218 more miles of travel than each successful DBD liver procurement. Current reimbursement policies poorly reflect increased surgeon travel (and time) expenditures between DCD and DBD liver offers.
\end{abstract}

KEYWORDS

deceased after brain death, deceased after circulatory death, donation, liver, reimbursement, travel

\section{1 | INTRODUCTION}

Donation after circulatory death (DCD) liver transplantation (LT) has increased slowly in the United States over the past decade, from 409 transplants in 2008 to 764 in $2018 .{ }^{1}$ In part, this modest growth may be due to increased surgeon and center resource utilization for DCD LT compared to donation after brain death (DBD) LT, including lower procurement rates for DCD donors and higher center costs per successful procurement. ${ }^{2}$ Given that transplant surgeons generally determine liver offer acceptance, understanding surgeon incentives and disincentives is paramount. One variable that might influence surgeon decision-making is the increased time commitment required for each DCD liver procurement, a surrogate measure of the opportunity costs of pursuing these types of liver donors. Accordingly, 


\begin{tabular}{|c|c|c|c|}
\hline & $\begin{array}{l}\text { DCD liver offer } \\
(n=309)\end{array}$ & $\begin{array}{l}\text { DBD liver offer } \\
(n=3039)\end{array}$ & $P$ value \\
\hline Offer acceptance, n (\%) & 48/309 (15.5) & 935/3039 (30.8) & $<.001$ \\
\hline $\begin{array}{l}\text { Successful procurement, } \mathrm{n} \\
(\%)^{\mathrm{a}}\end{array}$ & 23/48 (47.9) & $863 / 935$ (92.3) & $<.001$ \\
\hline $\begin{array}{l}\text { Distance travelled per } \\
\text { accepted offer, median } \\
(\mathrm{IQR}), \text { miles }\end{array}$ & $110(72,264)$ & $126(86,264)$ & .27 \\
\hline Air travel, n (\%) & $19 / 48(39.6)$ & $314 / 935$ (33.6) & .39 \\
\hline $\begin{array}{l}\text { Aggregate distance travelled, } \\
\text { miles }\end{array}$ & 10275 & 197299 & N/A \\
\hline $\begin{array}{l}\text { Aggregate travel distance } \\
\text { per successful procurement, } \\
\text { miles }^{\text {b }}\end{array}$ & 447 & 229 & $<.001$ \\
\hline
\end{tabular}

TABLE 1 Outcomes of DCD and DBD liver offers at Michigan Medicine, 2006-2017

Abbreviations: DBD, deceased after brain death; DCD, deceased after circulatory death; IQR, interquartile range.

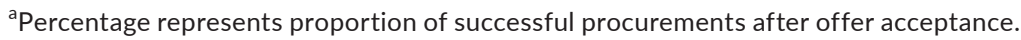

${ }^{\mathrm{b}}$ Computed by aggregate travel distance divided by the total number of livers successfully procured, by donor type. For DCD livers, this equates to 10275 miles divided by 23 successful procurements $=447$ miles travelled per successful procurement. For DBD livers, this equates to 197299 miles divided by 863 successful procurements $=229$ miles travelled per successful procurement.

we performed a center-level analysis of aggregate travel distance per successful DCD liver procurement compared to DBD liver procurement.

\section{2 | PATIENTS AND METHODS}

All consecutive liver offers made to Michigan Medicine from 2006 to 2017 were identified. Primary exposure was donor type (DBD or DCD). Primary outcome was the summative travel distance per successful liver procurement that resulted in LT. This was calculated by summing the total round-trip travel distances expended by donor type and dividing by the ultimate number of successful LTs. Secondary outcomes were liver offer acceptance and successful procurement proportion, and mode of transportation (ground versus flight). Wilcoxon rank-sum and chi-squared test were used to compare groups; significance was set at $P<.05$. Analyses were performed using STATA 15.1. This study was deemed exempt by the Michigan Medicine Institutional Review Board.

\section{3 | RESULTS}

During the study period, a total of 3348 potential liver offers were received by our center. Of these, 3039 (90.8\%) were DBD and 309 (9.2\%) were DCD. Liver offer acceptance was significantly lower for DCD ( $n=48 / 309,15.5 \%)$ than DBD ( $n=935 / 3039,30.8 \%)$ liver offers $(P<.001)$ (Table 1). After a procurement team was dispatched, successful procurement was significantly lower for DCD ( $n=23 / 48$, $47.9 \%)$ than $\operatorname{DBD}(n=863 / 935,92.3 \%)$ liver donors $(P<.001)$. Among accepted offers, including offers that did not result in successful procurement, median distance travelled was 110 miles (IQR 72-264 miles) for DCD livers and 126 miles (IQR 86-264 miles) for DBD livers $(P=.27)$. Proportion of air travel was not significantly different between the accepted DCD and DBD liver offers. Overall, 10275 miles were travelled for accepted DCD liver offers and 197299 miles were travelled for accepted DBD liver offers. This resulted in a mean 447 miles per successful DCD liver procurement and 229 miles per successful DBD liver procurement, a difference of 218 miles per successful liver procurement $(P<.001)$.

\section{4 | DISCUSSION}

On average, each successful DCD liver procurement required 218 more miles of travel than each successful DBD liver procurement. This difference was largely due to lower successful procurement proportion among DCD procurement episodes. Only $49.7 \%$ of DCD liver offers resulted in a liver procured for transplant, compared with $92.3 \%$ of DBD offers. Longer distances to travel suggest increasing surgeon time expenditure per successful DCD liver procurement.

Increasing distance and requisite time expenditures among DCD procurements may act as disincentives to DCD liver offer acceptance among transplant surgeons. This surgeon time and opportunity cost are compounded by increased costs to the transplant center for transportation, personnel, and recipient admissions during unsuccessful procurements that do not ultimately generate revenue via transplant reimbursement. ${ }^{2}$

Current compensation models poorly reflect the increased costs associated with lower successful procurement proportion among 
accepted DCD liver offers. In general, successful organ procurements are compensated at significantly higher levels than unsuccessful procurements. For instance, Michigan Medicine is given $\$ 4000$ per successful liver procurement from our organ procurement organization (OPO), but only $\$ 1250$ if unsuccessful. While this might be appropriate for DBD liver offers that have a high likelihood of success, this strategy might not be appropriate for DCD liver offers and further deter DCD offer acceptance. Additional work is needed to align surgeon/center costs and reimbursements with the increased resources expended for each successful DCD LT. This may include negotiating better standby surgeons' fees from OPOs or working with hospitals to create a pre-admission unit which is free for patients on "standby" waiting for a DCD liver offer.

This study is limited by its retrospective nature and generalizability as a single-center study. Donor characteristics and their effects on liver offer acceptance are unknown, as are the dispositions of other organs during procurement episodes. Nonetheless, our findings support the growing evidence of increased surgeon and center resource utilization during DCD liver procurements. Implementation of compensation models that adjust for lower successful procurement proportion and increased nonmonetary costs of DCD liver procurements must be addressed to support expansion of DCD LT. Further research is needed to characterize surgeons decision-making regarding DCD liver offer acceptance or refusal.

\section{CONFLICT OF INTEREST}

None.

\section{AUTHORS' CONTRIBUTIONS}

John Montgomery and Lisa McElroy: Contributed to concept/design, data analysis/interpretation, statistics, drafting article, critical revision of article, and approval of article; Alexandra Highet: Contributed to drafting article, critical revision of article, and approval of article; and Michael Englesbe and Mark Hobeika: Contributed to concept/ design, critical revision of article, and approval of article.

\section{ORCID}

John R. Montgomery (iD https://orcid.org/0000-0001-7751-231X

Mark J. Hobeika iD https://orcid.org/0000-0002-7444-7097

Lisa M. McElroy iD https://orcid.org/0000-0003-2366-2579

\section{REFERENCES}

1. Organ Procurement and Transplantation Network National Data. 2019;https://optn.transplant.hrsa.gov/data/view-data-reports/natio nal-data/. Accessed December 15, 2019.

2. Lindemann J, Dageforde LA, Vachharajani N, et al. Cost evaluation of a donation after cardiac death program: how cost per organ compares to other donor types. J Am Coll Surg. 2018;226(5):909-916.

How to cite this article: Montgomery JR, Highet A, Hobeika MJ, Englesbe MJ, McElroy LM. Going the distance for procurement of donation after circulatory death livers for transplantation-Does reimbursement reflect reality?. Clin Transplant. 2020;34:e13780. https://doi.org/10.1111/ctr.13780 